\title{
STATISTICAL ANALYSIS OF THE WATER LEVEL OF HUANG HE RIVER (YELLOW RIVER) IN CHINA
}

\author{
WANG Bo ${ }^{1}$, Zlatinka I. Dimitrova ${ }^{2}$, \\ NiKOLAY K. VITANOV ${ }^{3,4 *}$ \\ ${ }^{1}$ China-Central and Eastern Europe International Science and Technology \\ Achievement Transfer Center, Ningbo University of Technology, 201 Fenghua \\ Rd., Jiangbei Qu, Ningbo Shi, Zhejiang Sheng 315000, China \\ ${ }^{2}$ G. Nadjakov" Institute of Solid State Physics, Bulgarian Academy of \\ Sciences, Blvd. Tzarigradsko Chaussee 72, 1784, Sofia, Bulgaria \\ ${ }^{3}$ Institute of Mechanics, Bulgarian Academy of Sciences, Akad. G. Bonchev \\ Str., Bl. 4, 1113 Sofia, Bulgaria \\ ${ }^{4}$ Institute for Climate, Atmosphere and Water Research, Bulgarian Academy \\ of Sciences, Blvd. Tsarigradsko Chaussee 66, 1784 Sofia, Bulgaria
}

[Received: 19 February 2019. Accepted: 13 May 2019] doi: 10.7546/JTAM.49.19.02.04

\begin{abstract}
Very high water levels of the large rivers are extremely dangerous events that can lead to large floods and loss of property and thousands and even tens of thousands human lives. The information from the systematical monitoring of the water levels allows us to obtain probability distributions for the extremely high values of the water levels of the rivers of interest. In this article we study time series containing information from more than 10 years of satellite observation of the water level of the Huang He river (Yellow river) in China. We show that the corresponding extreme values distribution is the Weibull distribution and determine the parameters of the distribution. The obtained results may help for evaluation of risks associated with floods for the population and villages in the corresponding region of the Huang He river.
\end{abstract}

KEY WORDS: extreme events, water level, Huang He river, Weibull distribution

\section{INTRODUCTION}

Complexity and nonlinearity of the dynamics are important features of numerous natural and social systems [1-5]. Several examples of models of such systems are the models of meteorology based on the Lorenz equations [6,7], social dynamics models [8-12], population dynamics models based on the Lotka-Volterra equations and their

\footnotetext{
*Corresponding author e-mail: vitanov@imbm.bas.bg
} 
generalizations [13], turbulence theory [14-16], theory of nonlinear waves [17-19], etc.. The model equations of the fluid mechanics systems are nonlinear and thus the mechanics of flows is a large source of nonlinear problems and models [20-22]. Because of this research in fluid mechanics is an area of extensive application of the methods of nonlinear dynamics and the theory of chaos [23-26]. In this article we shall apply the methodology of the theory of extreme values to a problem from the research on the changing water levels of large rivers. As the number of floods increase in the last years the research on the water levels intensifies as the large rivers can cause large losses of property and human lives. In this research one uses the methods of the nonlinear time series analysis and extreme values theory [27-30]. These methods [31-34] and their combination [35] are very effective for analysis of time series and for description of many extreme events in Nature and society.

The global warming is another important reason for the research interest ion water levels. The global warming that leads to more rains and increasing of the levels of water that can cause floods and damages of houses, cities, ports and people. This was the reason for the begin of our research on statistical description of atmosphere and hydrological time series and study of the extreme values of these series [21,36, 37]. Below we shall study time series for the water level in for Huang He river in China. The Huang He (Yellow river) is the second longest river in Asia, after the Yangtze River, and the sixth longest river system in the world at the estimated length of $5464 \mathrm{~km}$. The total drainage area of the Huang He river is about 752,546 square kilometers. The Huang He river was very important for the ancient and contemporary Chinese civilization. In the history and in the more recent times frequent devastating floods and course changes of the flow of the river have been observed. These floods and changes are produced by the continual elevation of the river bed. The waters of the Huang He river are responsible for large catastrophes including the only natural disasters in recorded history to have killed more than a million people: the flood from 1332 - 33 that killed 7 million people; the flood from 1887, which killed between 900,000 and 2 million people, and the flood from 1931 that killed between 1 and 4 million people. Because of all of this the monitoring of the water level of the Huang He river and the study of the probabilities for extreme values of this level are important and even vital for the people of the regions along the river.

The organization of the text below is as follows. In Section 2 we describe the studied time series. In Section 3 we apply the extreme values theory to the time series and calculate the parameters of the extreme value distributions for the water level of the Huang He river. Several concluding remarks are summarized in Section 4. 


\section{The TIME SERIES}

The studied time series - Fig. 1(a) are obtained from the $\mathrm{x}$ database [38]. The water levels are measured on the base of satellite observation of the Hung He river from 21.07.2008 till 02.02 .2019 at longitude $115.1682^{\circ} \mathrm{E}$ and latitude $35.4222^{\circ} \mathrm{N}$. The satellite measurement of the water level is performed one time per 10 days. We observe significant changes in the water level of the river and the largest deviation from the average level of the river is more than two meters which is a large value and requires attention.

The time series from Fig. 1 show that in the period of measurement of more than 10 years the water level of Huang He river had several such larger deviations (of about two meters) from its average value. Another interesting observation is the decrease of the level of the river approximately in the middle of the interval of the observation (from 2014). This decrease leaded to decreasing height of the maximum water levels: in the first half of the observation period the maximum level was about
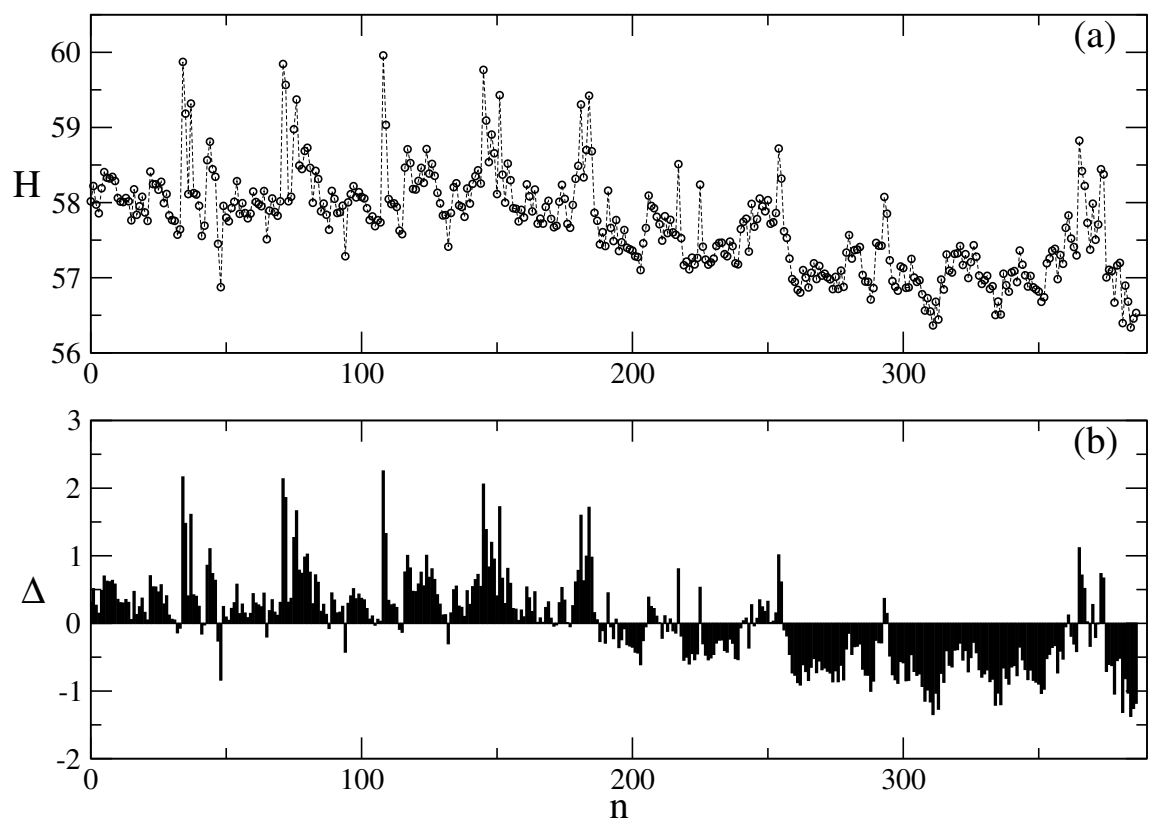

Fig. 1. Time series of the water level of the Huang He river (Yellow river) (Figure (a)) and for the deviation of the height of the water from its average value for the period of the records (Figure (b)). Horizontal axes: $n$ is the number of observation. Vertical axes: $H$ is the height of the water level in meters ( 0 is the sea level); $\Delta$ is the deviation of the height of the water (in meters) from the average height of the water for the period of the records. 
60 meters (height over the sea level). In the second part of the period of observation the maximum water level does not exceed 59 meters. This decrease of the water level can be seen also in the relationship $\Delta(n)$ in Fig. 1(b). where at the right-hand side of the figure the water level in the larger part of the time is below the average water level. We shall not discuss the reason for this drop of the water level. From the point of view of the extreme values theory the drop of the water level will lead to changes of the parameters of the extreme value distribution for the values of the water level. The the probability for extreme large water level (that can lead to large floods) will change.

Figure 2(a) shows the histogram and Fig. 2(b) shows the cumulative histogram connected to the studied time series. In Fig. 2(a) we clearly observe presence of extreme values of the water level (far right-hand side of the figure). Another interesting property of the histogram is presence of two areas of increased probability and area of decreasing probability (between 57.5 and 57.7 meters) of the water level of the
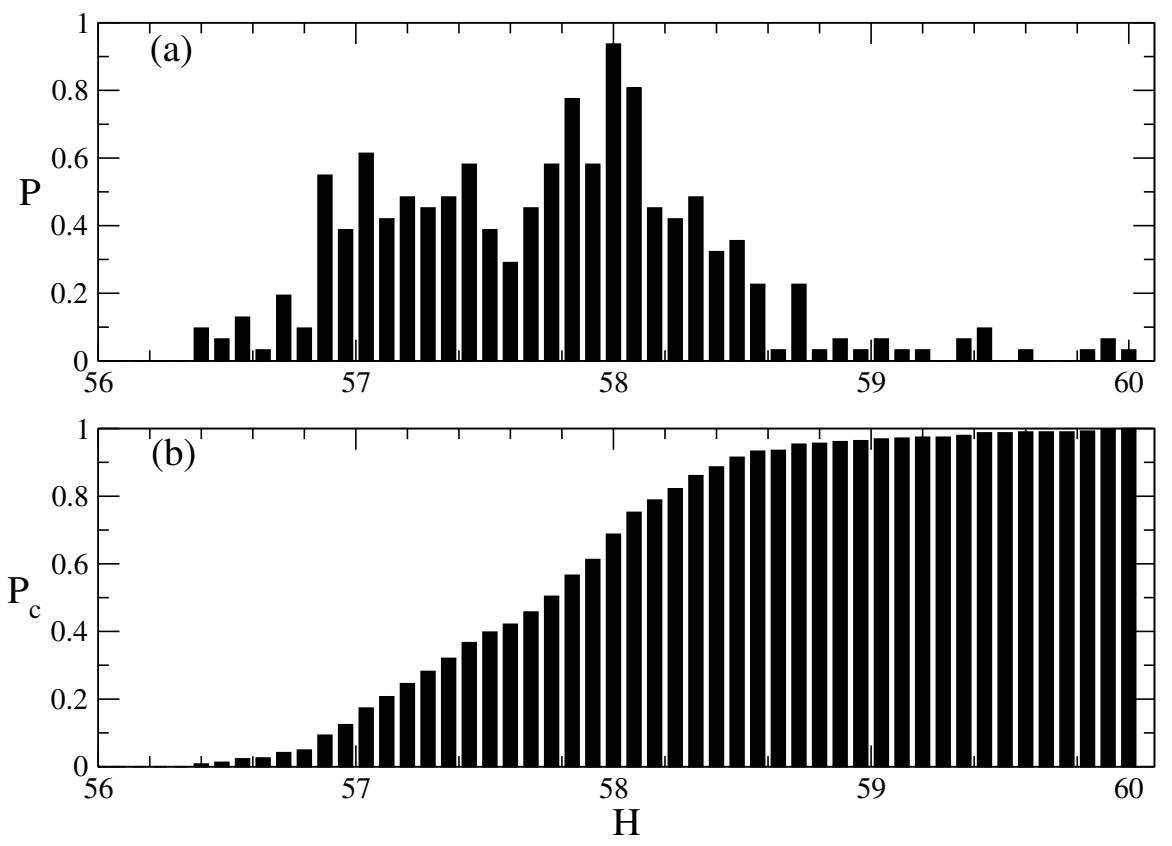

Fig. 2. Histogram (Figure (a )) and cumulative histogram (Figure (b)) for the time series of the water level of the Huang He river (Yellow river). Horizontal axes: $H$ is the height of the water level in meters ( 0 is the sea level). Vertical axes: $P$ : probability that the water level will be in the corresponding interval of values. $P_{c}$ : cumulative probability that the water level will be in the corresponding interval of values $P_{c}$. 
Huang He river. This corresponds to the drop of the water level of the river. The cumulative histogram in Fig. 2(b) shows that the water level of the Huang He river newer dropped below $56.4 \mathrm{~m}$ for the period of observation.

\section{Extreme Value Distributions}

The analyzed time series (Fig. 1(a)) can be divided into two approximately stationary parts separated by a small region of non-stationary data. We shall consider the two approximately stationary regions. We shall connect the water level of the river to a random variable. An example for realization of a random variable are the temperatures at a given place within a day. If we take the maximum daily temperature, we can construct time series for the maximal daily temperatures. We shall treat in similar way the time series for the water level. The water level rises and falls forming a wave-like structure. We shall take the water level for every such structure as a realization of a random variable and we shall investigate the time series for the maxima of these variables, i.e. the time series for the maximum wave heights.

Further we shall assume that the obtained maxima of the water level time series are maxima of a stationary sequence of random variables $\left\{H_{k}\right\}, k=1, \ldots, K$. We are going to study the limit distribution of these time series. In order to choose the appropriate methodology for this study we have to account for the following important fact namely that the limit distribution does not need to be the same as the distribution of the maxima of the associated independent sequence of random variables $\left\{\tilde{H}_{k}\right\}$, $k=1, \ldots, K$ with the same marginal distribution as $\left\{H_{k}\right\}$. Sometimes extreme values tend to occur in clusters and we have to quantify this tendency for the studied time series. This can be made by the extremal index $\theta$ which measures the tendency of the extreme values to occur in clusters. The mean size of the clusters of extreme values is $1 / \theta$. Thus if $\theta=1$, the mean size of the clusters of extreme values is 1 which means that there is no clustering of the extreme events (we have a single extreme value and the values in the time series that are around this extreme value are not extreme values). In this case the parameters of the extreme value distributions for the stationary sequence $\left\{H_{k}\right\}$ and for the independent sequence $\left\{\tilde{H}_{k}\right\}$ are the same.

We shall consider two kinds of estimators for $\theta$.

\section{The runs estimator}

$$
\hat{\theta}_{n}^{R}(u, r)=\frac{\frac{1}{n-r} \sum_{i=1}^{n-r} \mathbf{1}\left(H_{i}>u, M_{i, i+r} \leq u\right)}{\frac{1}{n} \sum_{i=1}^{n} \mathbf{1}\left(H_{i}>n\right)},
$$

where 
- $H_{i}, i=1, \ldots, n$ is the sample (e.g., the time series which are approximately stationary);

- $u$ is the threshold;

- $r$ is the run length;

- $M_{i, i+r}$ is the maximum value within the segment $H_{i+1}, \ldots, H_{i+r}$;

- 1 means that 1 is added to the corresponding sum if the condition in the () is fulfilled. Otherwise 0 is added to the corresponding sum.

2. The interexceedance times estimator

$$
\hat{\theta}_{N}(u)=\frac{2\left[\sum_{i=1}^{N-1}\left(T_{i}-1\right)\right]^{2}}{(N-1) \sum_{i=1}^{N-1}\left(T_{i}-1\right)\left(T_{i}-2\right)},
$$

where

- $N$ is the number of exceedances of the threshold value $\mathrm{u}$ and let these exceedances happen at times $1 \leq S_{1} \leq \leq S_{N}$;

- The interexceedance times are $T_{i}=S_{i+1}-S_{i}$.

We note that the interexceedance estimator is appropriate when the maximum interexceedance time is larger than 2 .

The application of the two estimators of $\theta$ to our time series shows that for the time series at the initial part of the period of observation (below the drop of the water level) The $\theta$-index is about 0.25 up to threshold level of 57 meters and then $\theta$ begins to increase arriving at $\theta=1$ (no clasterization of the extreme values) at threshold level of 58.95 meters. For the case of the time series after the drop of the water level (right-hand side of the observed time series) the $\theta$-index is about 0.25 up to threshold level of 57.06 meters (almost the same threshold as above) and then the $\theta$-index begins to increase arriving at $\theta=1$ (no clustering of the extreme values) at threshold level of 57.8 meters.

Next we have to determine the kind of the distribution of the extreme values and to calculate the parameters of this distribution. The kind of the distribution can be determined on the basis of quantile-quantile plots. In order to make such plots we have to define the corresponding quantile function. 
Let us have a time series $H_{1}, H_{2}, \ldots, H_{n}$ with distribution function $F(x)=$ $P(H \leq x)$. Important property of $F(x)$ is the inverse of the distribution function: the quantile function $Q(p)$ :

$$
Q(p)=\inf [x: F(x) \geq p] .
$$

We shall use the quantile functions much in the text below.

On the basis of the series $H_{i}$ we can define the empirical distribution function $\hat{F}(x)=\frac{i}{n}$ if $x \in\left[H_{i}, H_{i+1}\right]$ and $H_{i}$ are ordered by increasing value. Then the empirical quantile function is

$$
\hat{Q}(p)=\inf [x: \hat{F}(x)>p] .
$$

Let us use the quantile functions in order to produce some quantile-quantile plots for our data. We consider first the class of exponential distributions

$$
F_{\lambda}(x)=1-\exp (-\lambda x),
$$
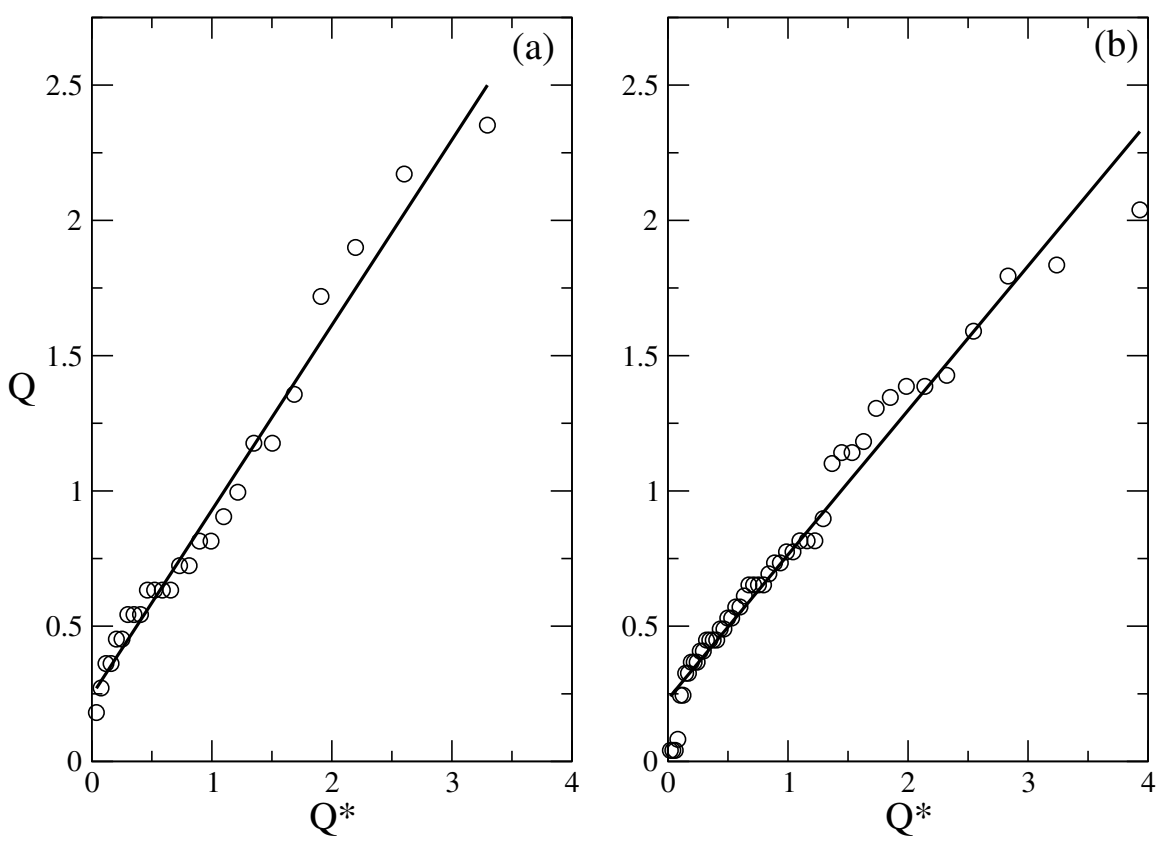

Fig. 3. Quantile-quantile plot. $Q^{*}$ : the standard exponential quantile. $Q$ : quantiles for the water level of the Huang He river. Figure (a): quantile-quantile plot for the first half of the time series. Figure (b): quantile-quantile plot for the second half of the time series (after the drop of the water level). 
where $\lambda$ is a parameter. The quantile function for this class of distributions is

$$
Q_{\lambda}(p)=-\frac{1}{\lambda} \ln (1-p)
$$

for $p \in(0,1)$. For $\lambda=1$ we have the standard exponential distribution and the quantile of distribution with parameter $\lambda$ is connected to the standard exponential quantile function as follows

$$
Q_{\lambda}(p)=\frac{1}{\lambda} Q_{1}(p), \quad p \in(0,1) .
$$

Let us construct the standard exponential quantile-quantile $(Q-Q)$ plot: $[-\ln (1-$ $p), \hat{Q}(p)]$ where we plot the quantile of the exponential distribution vs. the quantile of the observed time series. If the exponential distribution is a good fit to the experimental data $\hat{Q}(p)$ the standard exponential quantile plot will be close to a straight line. The results for the water level time series of the Huang He river are shown in Fig. 3.
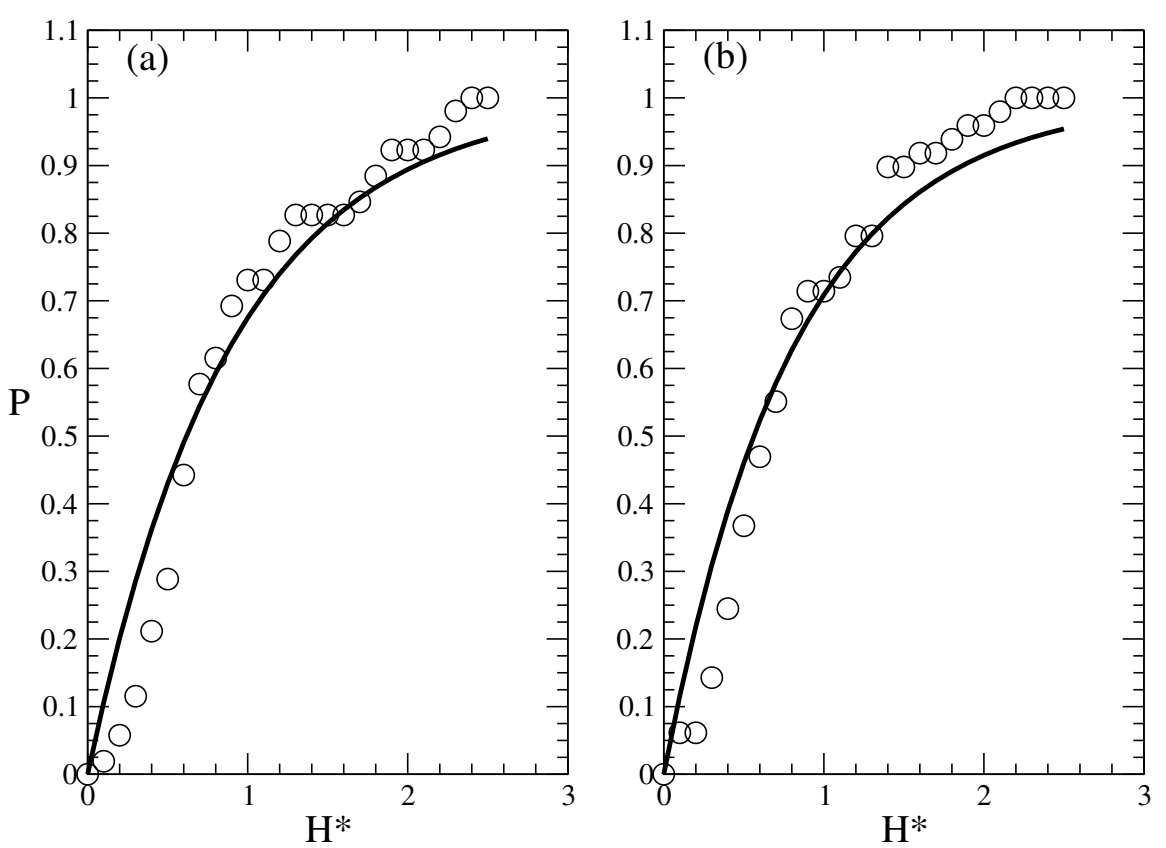

Fig. 4. The best fit of the extreme value distribution of the water levels of the Huang he river by the exponential distribution $F_{\lambda}(x)=1-\exp (-\lambda x) . H^{*}$ : relative height of the maximum over the threshold. $P$ : probability. The values of the parameter $\lambda$ are: Figure (a): $\lambda=1.123$; Figure (b): $\lambda=1.233$. 
We observe that the quantile-quantile plots are not close to a straight line for the case of large values of the quantiles, i.e. for th case of large values of the water levels. This means that the exponential distribution is not a good fit for the distribution of the extreme values for the both time series for the water levels of the Huang He river.

Figure 4 agrees with the conclusion that the exponential distribution is not a good fit for the distribution of the extreme water levels of the Huang He river. We see that the best fit exponential distribution is systematically lower than the values of the water levels for the case of large values of these levels. Thus we have to search for another distribution that will fit the data better.

One such distribution can be a member of the class of the extreme values distributions. There exist three kinds of extreme values distributions for i.i.d. random variables: Weibull, Gumbel and Frechet-Pareto. If some of these distribution is the extreme events distribution for the water level time series, the correspondent quantilequantile plot will be close to a straight line. We have obtained that that the Weibull distribution is the appropriate distribution for the maxima of the water level time
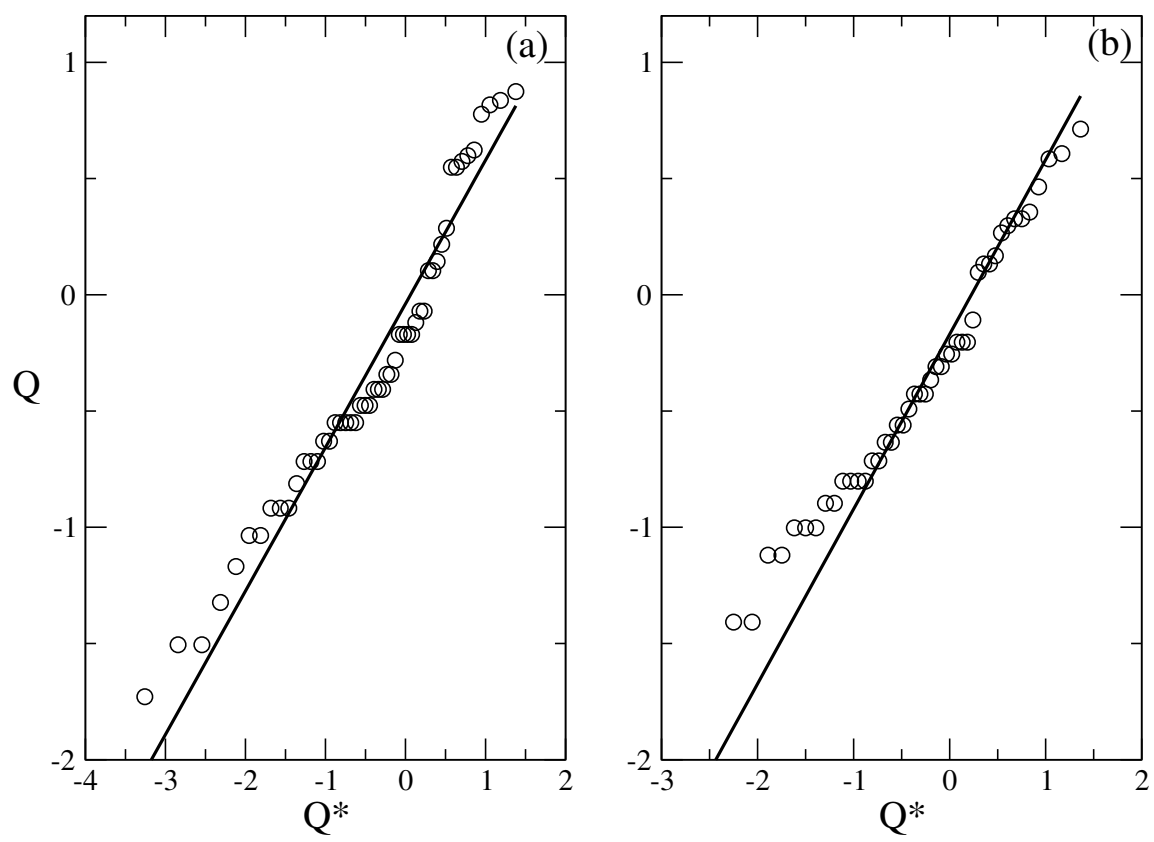

Fig. 5. Quantile-quantile plot. $Q^{*}$ : the quantile for the Weibull distribution. $Q$ : quantiles for the water level of the Huang He river. Figure (a): quantile-quantile plot for the first half of the time series. Figure (b): quantile-quantile plot for the second half of the time series (after the drop of the water level). 
series. The Weibull distribution is

$$
F_{W}=1-\exp \left(-\lambda x^{r}\right),
$$

where $x>0, \lambda>0$ and $r>0$. Probability density function for this distribution is $f_{W}=\lambda r x^{r-1} \exp \left(-\lambda x^{r}\right)$. The quantile-quantile plot of Weibull quantile vs. the quantile of the maxima of the water level time series is

$$
[\ln (-\ln (1-p), \ln (\hat{Q}(p)))] .
$$

This plot is shown in Fig. 5 for the two series of maximum values connected to the water level of the Huang He river. We observe that the quantile-quantile plot is close to a straight line for large values of $H^{*}$ and this closeness is especially seen at the right-hand side of Fig. 5(b). Thus our next step is to fit the Weibull distribution to the distribution of the maxima of the water level time series. The fit is shown in Fig. 6. We see that this fit is much better in comparison to the fit made on the basis of the exponential distribution (Fig. 4).
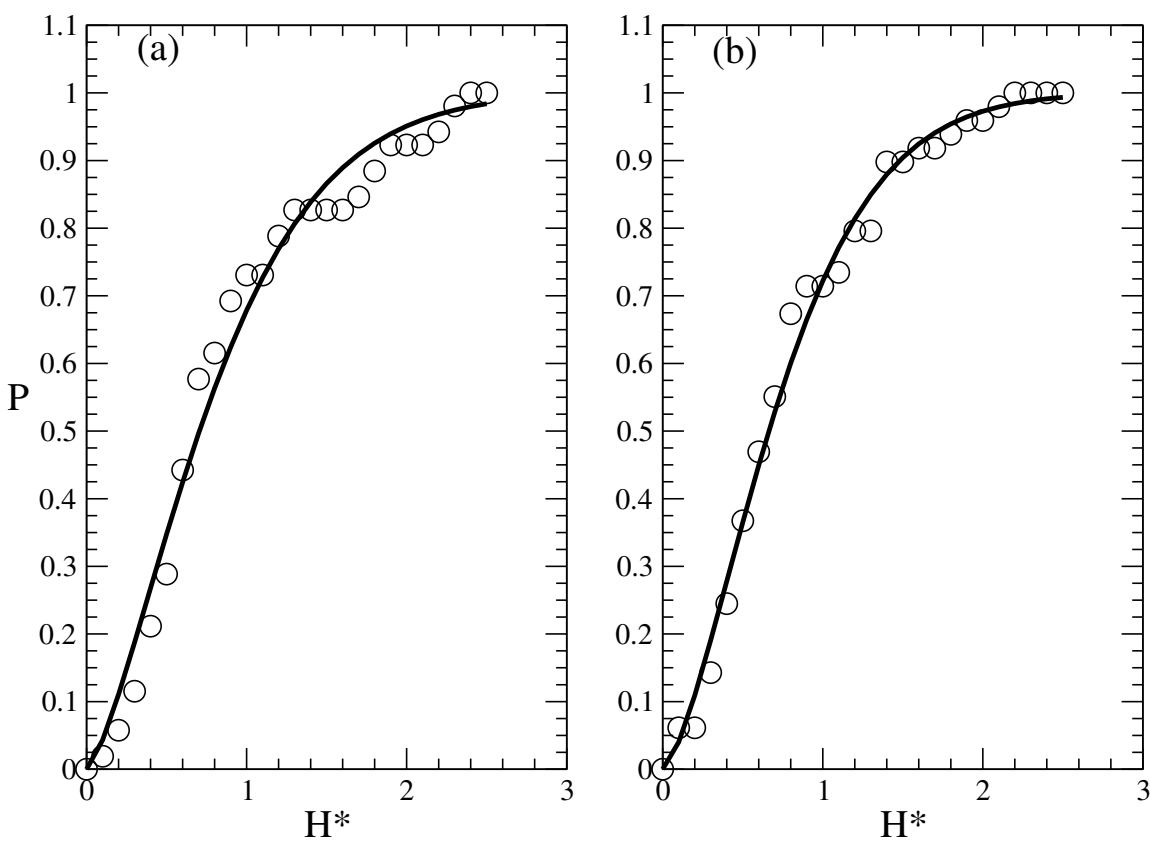

Fig. 6. The best fit of the extreme value distribution of the water levels of the Huang he river by the exponential distribution $F_{\lambda}(x)=1-\exp \left(-\lambda x^{r}\right) . H^{*}$ : relative heigh of the maximum over the threshold. $P$ : probability. The values of the parameter $\lambda$ and $r$ are: Figure (a): $\lambda=1.135, r=1.410$; Figure (b): $\lambda=1.280, r=1.494$. 
The obtained extreme value distribution for the water level of the Huang He river allow us to calculate the probabilities for very large values of these levels. These probabilities are summarized in Table 1.

Table 1. Probability of corresponding large value of the water level of the Huang He river (in $\%)$ before water level drop: $P_{1}$, and after the drop of the water level: $P_{2}$

\begin{tabular}{ccc}
\hline \hline Water level $(\mathbf{m})$ & $P_{1}$ & $P_{2}$ \\
\hline 61 & 0.170 & 0.00112 \\
62 & 0.0104 & 0.0000176 \\
63 & 0.000484 & 0.000000184 \\
64 & 0.0000176 & 0.00000000132 \\
65 & 0.000000516 & 0.00000000000672 \\
66 & 0.0000000123 & 0.0000000000000247 \\
\hline
\end{tabular}

The results show that before the water drop one could expect water level of $61 \mathrm{~m}$ one time in about 5880 days (one time in 16 years) and after the drop of the water level this probability has decreased to 1 time in 89285 days (one time in 245 years).

\section{CONCLUding REMARKS}

In this article we analyze time series containing values for the water level of the second largest river in China - The Huang He river. Our goals was to obtain the distribution for the extreme values of the water levels of the river around the point of the measurement of the water level. We observe a drop of the water level of the river and divide the time series for the water level into two parts: before the drop of the water level and after the drop of the water level. We obtain that the extreme values distribution for the corresponding time series is the Weibull distribution. We calculate the parameters of this distribution. On the basis of the obtained distribution it is easy to obtain the probability of extreme water levels. This may be of great help for the decision making about all the infrastructure, villages, town and cities that can be affected by such high water levels. We note that results are obtained on the basis of time series recorded in a given region of the river and the drop of the water level leaded to significant decrease of the probability of extreme large water levels and corresponding large floods.

\section{REFERENCES}

[1] Reggiani A., P. NiJkamp (eds.) (2000) "Complexity and Spatial Networks". Springer, Berlin, Germany.

[2] Rissanen J. (2007) "Information and Complexity in Statistical Modeling". Springer, New York, USA. 
[3] Vitanov, N. K. (2016) "Science Dynamics and Research Production. Indicators, Indexes, Statistical Laws and Mathematical Models”. Springer, Cham, Switzerland.

[4] Meyers, R. A. (2009) 'Encyclopedia of Complexity and Systems Science”. Springer, New York, USA.

[5] Kreimeyer M., U. Lindemann (2011) "Complexity Metrics in Engineering Design". Springer, Berlin, Germany.

[6] PAnchev S. (2000) "Theory of Chaos". Marin Drinov Publishing House of the Bulgarian Academy of Sciences, Sofia, Bulgaria.

[7] Panchev S., T. Spassova, N. K. Vitanov (2007) Analytical and numerical investigation of two families of Lorenz-like dynamical systems. Chaos Solitons \& Fractals 331658 - 1671.

[8] Treiber M., A. Kesting (2013) "Traffic Flow Dynamics: Data, Models, and Simulation". Springer, Berlin, Germany.

[9] Vitanov N. K., K. N. Vitanov (2018) Discrete-time model for a motion of substance in a channel of a network with application to channels of human migration. Physica A 509635 - 650.

[10] CHAN W.-K. (1990) "Theory of Nets: Flows in Networks". Wiley, New York, USA.

[11] Vitanov N. K., K. N. Vitanov (2016) Box model of migration channels. Mathematical Social Sciences 80108 - 114.

[12] Simon J. H (1999) "The Economic Consequences of Migration". The University of Michigan Press, Ann Arbor, MI, USA.

[13] Vitanov N. K., I. P. Jordanov, Z. I. Dimitrova (2009) On nonlinear dynamics of interacting populations: Coupled kink waves in a system of two populations. Commun. Nonlinear Sci. Numer. Simulat., 142379 - 2388.

[14] Boeck T., N. K. Vitanov (2002) Low-dimensional chaos in zero-Prandtl-number Benard-Marangoni convection. Phys. Rev. E 65037203.

[15] Vitanov N. K. (2000) Upper bounds on convective heat transport in a rotating fluid layer of infinite Prandtl number: Case of intermediate Taylor numbers. Phys. Rev. E 62 3581 - 3591 .

[16] Vitanov N. K. (2000) Convective heat transport in a fluid layer of infinite Prandtl number: upper bounds for the case of rigid lower boundary and stress-free upper boundary. European Physical Journal B 15349 - 355.

[17] Remoussenet M. (1993) "Waves Called Solitons”. Springer, Berlin, Germany.

[18] Vitanov, N. K., T. IVAnova, Z. I. Dimitrova(2017) On solitary wave solutions of a class of nonlinear partial differential equations based on the function $1 / \cosh ^{n}(\alpha x+\beta t)$. Applied Mathematics and Computation 315372 - 380.

[19] Vitanov N. K. Breather and soliton wave families for the sine-Gordon equation (1998) Proc. Roy. Soc. London A 4542409 - 2423.

[20] KAMBE T. (2004) "Geometrical Theory of Dynamical Systems and Fluid Flows" World Scientific, Singapore. 
[21] Kantz H., D. Holstein, M. Ragwitz, N. K. Vitanov (2004) Markov chain model for turbulent wind speed data. Physica A 342315 - 321.

[22] Godreche C., P. Manneville (eds.) (1998) "Hydrodynamics and Nonlinear Instabilities". Cambridge University Press, Cambridge, UK.

[23] Pedlosky J. (1987) "Geophysical Fluid Dynamics”, Springer, New York, USA.

[24] Vitanov N. K. (2000) Upper bounds on the heat transport in a porous layer. Physica $D, 136322$ - 339.

[25] Vitanov N. K. (2000) Upper bound on the heat transport in a layer of fluid of infinite Prandtl number, rigid lower boundary, and stress-free upper boundary. Phys. Rev. E 61 956 - 959.

[26] Velasco Fuentes O. U., J. Scheinbaum, J. Ochoa (2003) ”Nonlinear Processes in Geophysical Fluid Dynamics". Kluwer, Dordrecht, Netherlands.

[27] Kantz H., T. Schreiber (1997) ”Nonlinear Time Series Analysis”, Cambridge, Cambridge University Press, UK.

[28] Vitanov N. K., E. D. Yankulova (2006) Multifractal analysis of the long-range correlations in the cardiac dynamics of Drosophila melanogaster. Chaos, Solitons \& Fractals 28768 - 775.

[29] Coles S. (2001) "An Introduction to Statistical Modeling of Extreme Values". Springer, London, UK.

[30] Reiss R.-D., M. Thomas (1997) "Statistical Analysis of Extreme Values". Birkhäuser, Basel, Switzerland.

[31] CRYer J. D., K.-S. Chan (2008) "Time Series Analysis". Springer, New York, USA.

[32] Vitanov N. K., K. Sakai, I. P. Jordanov, S. Managi, K. Demura (2007) Analysis of a Japan government intervention on the domestic agriculture market. Physica A 382330 - 335.

[33] Mills T. C. (2011) "The foundations of Modern Time Series Analysis". Palgrave Macmillan, New York, USA.

[34] Vitanov N. K., K. Sakai, Z. I. Dimitrova (2008) SSA, PCA, TDPSC, ACFA: Useful combination of methods for analysis of short and nonstationary time series. Chaos Solitons \& Fractals 37187 - 202.

[35] Albeveiro S., V. Jentsch, H. Kantz (eds.) (2006). "Extreme Events in Nature and Society", Springer, Berlin, Germany.

[36] Vitanov N., N. Hoffmann (2009) On probability for rogue waves in the North sea. Compt. rend. Acad. bulg. Sci. 62187 - 194.

[37] Dimitrova, Z. I., N. P. Hoffmann (2012) On probability for extreme water levels of the river Elba in Germany. Compt. rend. Acad. bulg. Sci. 65153 - 160.

[38] Schwatke C., D. Dettmering, W. Bosch, F. Seitz (2015) DAHITI - an innovative approach for estimating water level time series iver inland waters using multimission satelite altimetry. Hydrology and Earth System Sciences 194345 - 4364. 